\title{
Troublesome crying in infants: effect of advice to reduce stimulation
}

\author{
Sheila McKenzie
}

Abstract

The observation that babies with troublesome crying improve quickly in hospital suggested that, if true, a common, quickly reversible, factor may operate. Histories from parents of such babies suggest that much work goes into trying to console them. It is hypothesised that this may lead to excessive stimulation and the improvement seen in hospital reflects a reduction in stimulation.

Two studies were undertaken. (1) Carers were asked to agree to randomisation of their infants to hospital or home management. Those at home were advised to reduce stimulation. A 10 point questionnaire was used to describe distress in mothers of subjects and age matched controls. (2) A randomised controlled study compared advice to reduce stimulation with an empathic interview using $a+5$ to -5 scale to chart change. In the first study too few subjects agreed to randomisation and thus a rigorous study to validate the observation could not proceed. There was good evidence, however, that crying improved. Results from the home group justified the second study. The median distress score for subjects was $7 / 10$ and for controls $3 / 10$ $(\mathbf{p}<0.001)$. In the second study at seven days, $18 / 22$ subjects given advice scored +2 or better on the change chart for crying, compared with $7 / 20$ of those who did not receive advice $(p<0.01)$. After the latter received advice $79 \%$ improved $(95 \%$ confidence interval 61 to $97 \%$ ). For babies under 12 weeks, the customary upper limit for a diagnosis of colic, 14/15 subjects advised improved compared with $6 / 12$ who were not advised $(p<0.02)$.

These studies have shown that infants with troublesome crying admitted to hospital seem to improve quickly as do those whose carers are advised to reduce stimulation.

There are two broad proposals to explain the problem of troublesome crying in infants. ${ }^{12}$ One proposal suggests an organic cause for a syndrome of excessive crying in infants of less than 3 months of age that is presumed to be caused by pain and excessive flatus, that is, colic. The second suggests a non-organic, developmental, or behavioural cause.

Because of the uncertainty of the cause there

Neonatal Unit, Rush Green Hospital, Dagenham Road, Romford,

Essex RM7 OYA

Correspondence to: Dr McKenzie.

Accepted 17 June 1991
, understandably, large numbers Although 20 years ago Brazelton urged mothers of babies with troublesome crying to 'offer lactose (at least hourly) to facilitate mobilisation of stomach gas', ${ }^{3}$ lactose intolerance is nowadays the front runner in the organic category of proposed causes of troublesome crying. There is no evidence to date, however, that either milk allergy or lactose intolerance is responsible for excessive crying. Taubman demonstrated in a group of symptomatic babies that a reintroduction of cows' milk after its elimination did not reproduce symptoms. ${ }^{4}$ It has yet to be shown in babies with colic that an increase in breath hydrogen excretion, suggestive of lactose intolerance, is not a secondary phenomenon. ${ }^{56}$ Hunziker and Barr have suggested that increased carrying is helpful in reducing crying in normal babies less than 3 months old and may prevent infant colic. ${ }^{7}$ Others suggest that a reduction in picking $\mathrm{up}^{8}$ or systematic ignoring ${ }^{9}$ are also helpful. Taubman concluded from a successful study of counselling parents about how to respond to infant crying that a major cause of colic was the misinterpretation of infant cries leading to ineffective responses. ${ }^{10}$

\section{Background and observations}

In 1986 a crying baby referral system was organised in Romford. Babies referred had no clinical features to suggest that any organic disorder was the cause of the principle symptom, inconsolable crying, which was causing considerable distress to carers. Some babies were admitted to hospital, mainly because it was felt that a carer might harm the child.

Most of the hospitalised babies' crying improved within a day or two. This suggested that there might be a common factor operating in hospital that was not operating at home. If that was identified then it might be possible to treat babies at home; it might also be possible to gain some insight into the cause of troublesome crying.

Also noticeable, both on history and on observation, was how hard the carers worked to try to stop the crying. Was it possible that this resulted in too much stimulation and that such infants' crying would be less troublesome in hospital because the nurses were not intervening in the same way?

\section{Purpose}

Two studies were undertaken:

STUDY 1

(a) To record biographical details and distress of carers attending the clinic.

(b) To discover if carers were willing to enter a randomised study of home versus hospital treatment to validate the observation that when babies with troublesome crying are admitted to 
hospital there is a clinically important reduction in crying.

(c) To examine the use of change rating charts.

(d) To discover whether the effect of advice to reduce stimulation at home was sufficient to justify a second study.

\section{STUDY 2}

To discover if advice to reduce stimulation of babies with troublesome crying managed at home was more effective than an empathic interview only.

\section{Methods for both studies}

Troublesome crying was defined as crying with which parents felt they could no longer cope and for which they sought help. Babies $<1$ year old were referred by health visitors and general practitioners. Consecutive babies were enrolled for both studies. Families were interviewed within 72 hours of referral in an office separate from outpatients. Only the interviewer and the family were present. This setting was designed to facilitate trust between the carers and paediatrician. General practitioners or community doctors had already assessed the baby to exclude physical illness.

\section{Biographical data}

The age at time of referral, position in family, length of time of troublesome crying, the pattern and length of crying or 'whingeing' (the local term for fussing) in the previous 48 hours, the carers' perception of the problem and the interventions already tried, and obstetric, feeding, family and social histories were recorded. The purpose of gathering this information was not only to describe the patient base but also to facilitate an empathic relationship between interviewer and carer(s).

\section{Distress score questionnaires}

Each mother (fathers did not always attend) was asked to answer a 10 point 'distress score' questionnaire (table 1). Five subgroups of items highlighted poor self esteem (items 1-3), feelings about the baby's manipulation (items 4,5 ), anger (items 6, 7), effect on health (items 8, 9), and other relationships (item 10). The questions had been understood by previous families in a pilot study.

Table 1 Distress questionnaire

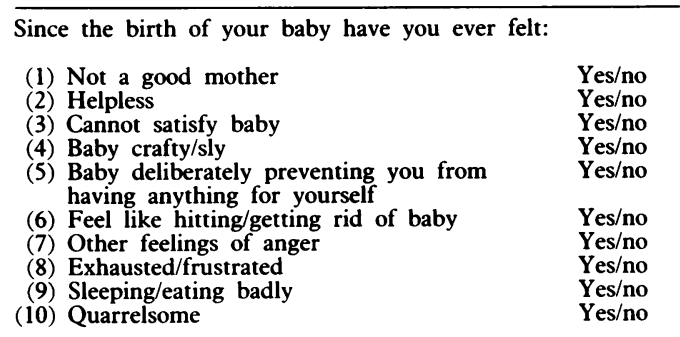

\section{Advice to reduce stimulation}

The carers were asked not to pat, wind, or lift their babies excessively. They were also asked to cuddle the baby gently, to distinguish between $\geq$ rocking (a relaxed 60 beat per minute swaying $\stackrel{3}{S}$ movement of baby in arms or on chest) and 0 jiggling ( $\mathrm{a}>140$ beat per minute jerking move- $\omega$ ment of baby on knees or in arms) and to avoid $\frac{\rho}{\partial}$ the latter. They were also to reduce stimulation from loud music, rattling toys, etc, and not to $\overrightarrow{=}$ intervene during the early part of a sleep when $\stackrel{f}{\rightarrow}$ the baby may vocalise and appear restless-an explanation of active sleep was given. An $\frac{\bar{\sigma}}{\bar{D}}$ assurance was given that a certain amount of $\frac{\bar{D}}{\vec{D}}$

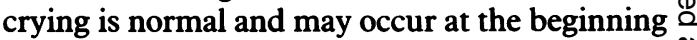
of sleep. Finally, the carers were given permis- ॐ sion to leave their infants if they felt that $\vec{\circ}$ because of the crying they could no longer tolerate their being close.

\section{Change rating charts}

A change rating chart was used to record the change in both the crying and in the mothers' ?

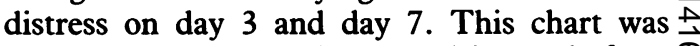
simply a line divided into equal intervals from +5 (better) through 0 (no change) to -5 은 (worse). Carers were asked to indicate on the $\vec{\nabla}$ chart a score which best reflected change in $\mathbb{D}$ both crying and distress from the time of the $\Phi$ first interview. A score of +2 or better or -2 or worse was considered a clinically important $\stackrel{\Phi}{\square}$ change.

\section{STUDY 1}

Thirty one subjects were enrolled and 31 age matched controls were randomly selected from well baby clinics. The median age was 10 weeks $\stackrel{\varnothing}{\varnothing}$ (range 3 weeks -10 months) and for the subjects $\vec{\overrightarrow{ }}$ the median length of crying was 8 weeks (range 3 1 week-8 months) before the first interview. There was no difference between subjects and controls either in the position in the family or in the type of feed offered. The median number of hours crying or whingeing during the 24 hour $\frac{5}{3}$ period before the interview was 12 hours $(4-21)$ for the subjects and less than 3 hours $(0-12) \frac{2}{3}$ for controls. Two of the controls admitted to troublesome crying.

Total distress scores for each patient (out of a possible total of 10) were calculated from the distress questionnaires and scores for subjects $\sigma$ and controls were compared by Wilcoxon rankN sum test for paired values. As the subgroups of N selected items were of unequal size, an alter- $\sigma$ native score was calculated in which one or

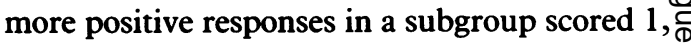

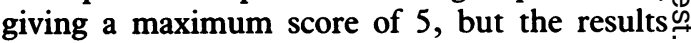
were very similar and will not be reported.

The median score for subjects was 7 (range음 3-10) and for controls was 3 (range $0-3) \stackrel{\mathbb{\Omega}}{\Omega}$ $(\mathrm{p}<0.0001)$. The median difference within $\underset{\Omega}{\mathbb{Q}}$ patient-control pairs was +3 (range +7 to -3 ) $(\mathrm{p}<0.0001)$.

Subjects were asked to agree to randomisationo or to elect for their babies to be managed either in hospital or at home. Those at home were? asked to reduce stimulation of their babies and to keep diaries of crying times. Those in 
hospital stayed for three days. Only one mother of 13 hospitalised babies stayed with her baby. As expected too few parents agreed to randomisation and so a formal comparison of hospital and home management could not proceed. The babies in hospital did however cry much less (2.5 hours/day compared with a reported 12 hours before admission) and this was concordant with the improvement in the change rating scores. This improvement was sustained after discharge.

Half of those managed at home did not return diaries or returned diaries which they admitted were incomplete. Those managed at home also improved significantly.

This study showed that a randomised comparison between hospital and home care would be impractical, as was the use by home carers of detailed crying diaries. However, it was established that a combination of interview and advice to reduce stimulation suggested a substantial improvement in babies managed at home. The second study was undertaken to examine the part played by the giving of advice.

\section{STUDY 2}

This examined whether advice to reduce stimulation helped more than just an empathic interview.

\section{Methods}

In the first study, $80 \%$ of babies with troublesome crying managed at home achieved change scores of +2 or more at day 7 . It was considered that a difference of $\mathbf{4 0 \%}$ in this proportion between an advice and an interview only group would be of practical importance. To demonstrate this with a type 1 error of 0.01 and a type 2 error of $0 \cdot 2$, it was planned to enrol 30 babies in each arm of a randomised trial. It was also planned to make a single interim analysis when 20 babies had been enrolled in each arm. As before, a change score of +2 or better was counted as a positive response, and the proportions of such responses were compared using $\chi^{2}$ testing with Yates's correction for continuity.

Carers were interviewed as in the first study. All carers were given an optimistic outlook. All agreed that information about their babies' crying and its management could be used in a research project. It was considered ethically acceptable that families should not be asked to agree to randomisation as knowledge of this may have prejudiced the quality of the information gathered; all eventually received the same treatment. Patients were randomised into two groups using random numbers. Each subject of the 'empathy' group was asked to fill in a diary of crying times and interventions for a week. This diary record was not for formal analysis; it was explained to the families that it would provide a background for advice in seven days time. The other group was advised to reduce stimulation. All were given a change rating chart and knew they would be contacted by telephone by someone who was not involved in the study and who would therefore not be able to advise.
All were contacted by telephone on days 3,7 , 10,14 , and at one month by an observer, usually a house officer, who did not know to which group infants had been assigned. The observer was given written instructions about how to conduct the telephone conversation with examples of questions and responses; he was asked to introduce himself by first name only and to request the results of the change scores on that day. Observers were to avoid conversation about anything else. All carers were contacted on the same days by the interviewer who did not know the results of the change scores. 'Empathy' group subjects returned on the afternoon of day 7 and were given the same advice about reducing stimulation as the other group.

\section{Results}

When 45 infants were enrolled an interim analysis was made. Three babies were not included: one requested hospital admission and two were not available on the telephone. The median age was 10 weeks and the median distress score was 7 (4-10).

The results of the change scores for crying on day 7 are shown in fig 1 and all results are summarised in table 2 . Three families were not available after seven days for follow up. The proportion of positive responses, both for babies' crying and for mothers' distress, was significantly higher in the 'advice' group on days 3 and 7. At this point the empathy group also received advice, and the proportion of

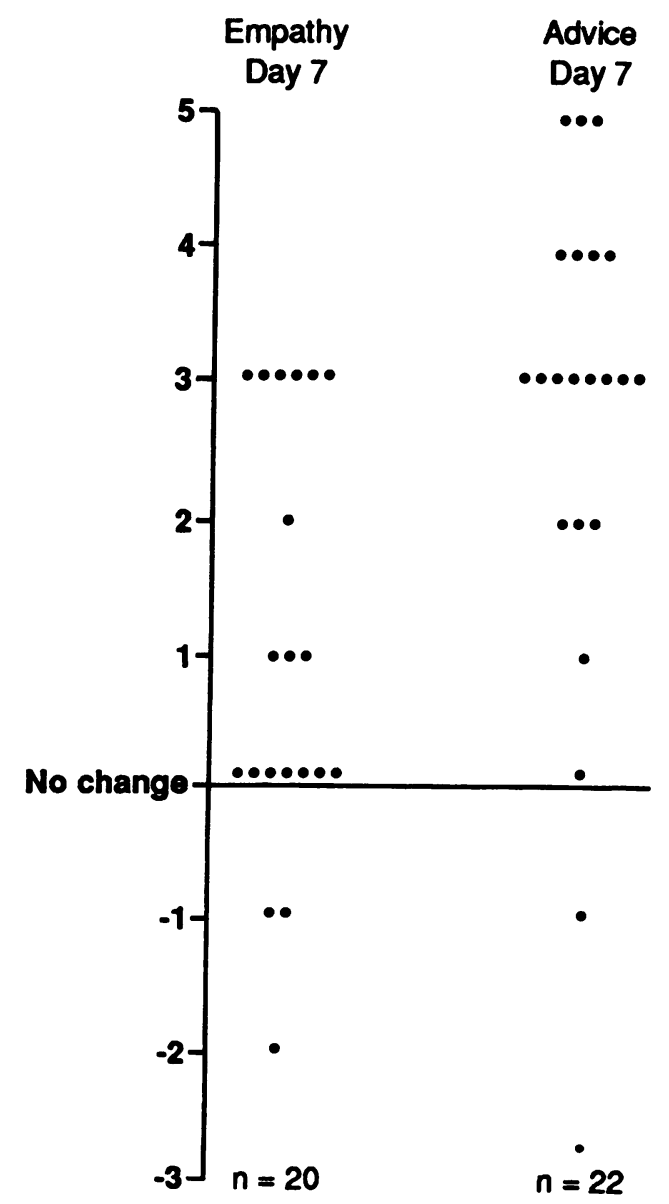

Advice

Figure 1 Change in crying seven days after start of study. 
positive responses rose to a level similar to that in the 'advice' group (fig 2). The results appeared sufficiently clear cut that no further subjects were enrolled. Thirty nine families were available for follow up one month after receiving advice and their change scores showed $36 / 39$ mothers scored +2 or better $(22=+5)$ and $30 / 39$ babies scored +2 or better $(19=+5)$.

Athough it was not the original intention to enrol enough babies for an analysis of separate subgroups, the opportunity was taken to examine the outcome for those aged below 12 weeks. This is the age many believe is the upper limit for colic. Twenty one of 27 carers with babies 12 weeks old or less offered a cause of the crying as

Table 2 'Empathy' and advice management. Numbers of babies and mothers who scored +2 or better on the change rating charts for baby's crying and mother's distress. The empathy group received advice on day 7

\begin{tabular}{lrll}
\hline & Empathy (\%) & Advice (\%) & $p$ Value \\
\hline Crying: & & & \\
Day 3 & $4 / 20(20)$ & $14 / 22(64)$ & $<0.02$ \\
Day 7 & $7 / 20(35)$ & $18 / 22(82)$ & $<0.01$ \\
Day 10 & $13 / 19(68)$ & $15 / 20(75)$ & $>0.5$ \\
Day 14 & $16 / 19(84)$ & $16 / 20(80)$ & $>0.5$ \\
Mother's distress: & & & \\
Day 3 & $4 / 20(20)$ & $15 / 22(68)$ & $<0.01$ \\
Day 7 & $6 / 20(30)$ & $19 / 22(86)$ & $<0.001$ \\
Day 10 & $14 / 19(74)$ & $17 / 20(85)$ & $>0.5$ \\
Day 14 & $16 / 19(84)$ & $16 / 20(82)$ & $>0.5$ \\
\hline
\end{tabular}

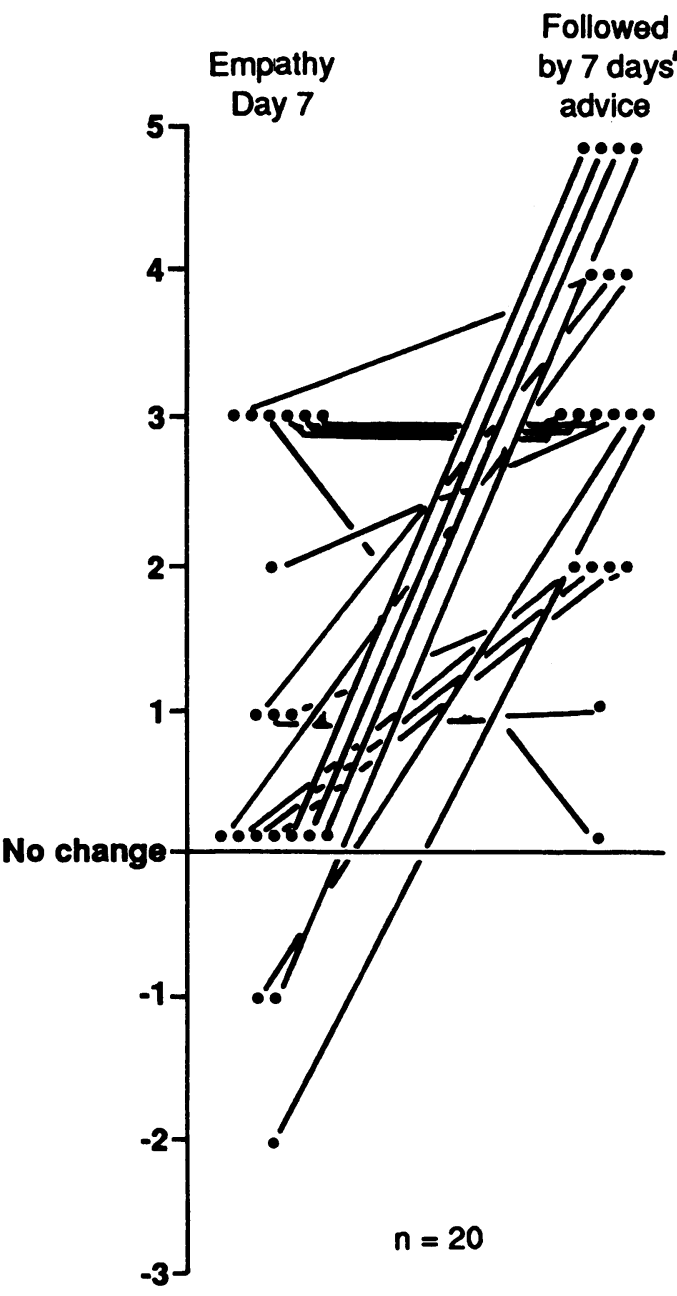

Figure 2 Change in crying in 'empathy' group after a further seven days advice; $79 \%$ (95\% confidence interval 61 to $97 \%$ ) improved. colic or wind compared with nine of 15 with older babies $\left(\chi^{2}=0.22 ; p=>0.5\right)$. Items 4 and 5 of the questionnaire-perception of baby's wilfulness-were examined in babies older and younger than 12 weeks. The younger babies scored 23 out of a possible total score of 54 compared with a score of 9 out of a possible 30 for older babies $\left(\chi^{2}=0.82 ; p=>0 \cdot 1\right)$. In babies less than 12 weeks six of 12 babies in the empathy group score +2 or better after seven days compared with 14 or 15 in the advice group $\left(\chi^{2}=4 \cdot 4 ; p<0 \cdot 02\right)$

\section{Discussion}

Implicit in the term 'troublesome crying' is the distress a baby's crying causes carers. In any study of this problem some measure of carers' distress should be made so that comparisons with future studies are possible. The 10 point distress score used in this study separated subjects and controls with very little overlap. It may well be that the completing of the questionnaire has a value of its own as it validates carers' feelings.

The observation that some babies with troublesome crying improve after hospital admission has been made before. ${ }^{11}$ In the present study, as expected, too few carers agreed to randomisation for home or hospital management to encourage the undertaking of a rigorous study to validate properly the observation. Nevertheless, the results indicated that babies nursed in hospital improved quickly and this was sustained after discharge.

The results of the second study which compared the effect of an empathic interview with such an interview augmented by specific advice to reduce stimulation indicate that advice is helpful. Whether this is any better than any other advice or intervention is not known. There is of course no evidence that stimulation was reduced. The effect of the empathic interview alone cannot be measured from this study as no group was identified who would be followed up in the community. Such a study was beyond the scope of this project.

The findings of both studies do not support the hypothesis that most troublesome crying is due to a primary organic cause. As lactose intolerance appears to be an important candidate for such an organic cause it would be interesting to undertake hydrogen breath studies in a group o of babies with troublesome crying admitted to $N$ hospital under conditions similar to this study. N If the crying stops quickly without a change in 0 diet a return of breath hydrogen to normal would suggest that previous raised levels are secondary, perhaps due to increased gut $\stackrel{\mathcal{D}}{\rightarrow}$ motility. ${ }^{12}$ This might result from sympathetic stimulation caused by the effort and exercise of excessive crying.

The diagnosis within the community of ' 3 - 8 month colic' is based on the observation that crying with wind improves at this age. Levine 8 describes changes in caretaking practices in infancy which move from emphasising survival to the transmission and maintenance of cultural values and goals. ${ }^{13}$ It is possible that around 3 months, carers respond to crying differently 
than they did before this and this may be the reason the crying stops. Indeed, Brazelton advises parents of 3 month old infants not to respond to cries and activity during waking periods and to 'condition' infants to use their own resources for sleep as early as possible. ${ }^{14}$ In other words, parents may worry that their baby has an organic cause for the crying in the first few months, may work hard to soothe the crying and to reassure themselves that the baby is healthy, and then make less of an effort once they perceive the baby as healthy and perhaps 'wilful'. In this study carers differed from this model. They were distressed by the crying and, having already seen their own doctor and been reassured that no organic cause could be found, sought help. Both colic and wilfulness were described throughout the age range studied. The advice worked for babies less than 3 months just as well as for those older. Thus the crying may well have a similar root.

At first sight this study's findings may appear contradictory to the findings of Hunziker and Barr who indicated that increased carrying reduced crying. ${ }^{7}$ Their study, however, considered babies' 'normal' crying. Pederson has indicated that rocking is helpful in reducing activity but there has been no rigorous examination of the effect of rocking after crying has become troublesome. ${ }^{15}$ In this study, parents were asked to hold the babies still or rock slowly in a quiet room. It was explained that some crying is normal ${ }^{2}$ and may occur as the baby falls asleep. Many parents reported that they felt that being given the permission to leave the baby if they could not cope was a great step forward. It was not the purpose of this study to examine the cause of troublesome crying but it is tempting to speculate that a carer's excessive jiggling, patting, and winding and other similar manoeuvres in response to crying may be inappropriate. If this is the response to crying at a time when a baby cries or vocalises when falling into active sleep it may prevent progression into quiet sleep and interfere with the development in a very young infant of a normal sleep pattern. Perhaps the encouragement of carrying and very gentle rocking in a peaceful environment in place of more stimulating interventions would prevent troublesome crying in the first place.

I am grateful to Professor Michael Healy for advice abou statistical methods and study design and to the many junior doctors who were observers.

1 Geertsma MA, Hyams JS. Colic-a pain syndrome of infancy Pediatr Clin North Am 1989;36:905-19.

2 St James-Roberts I. Persistent crying in infancy. $f$ Child Psychol Psychiatry 1989;30:185-95.

3 Brazelton TB. Crying in infancy. Pediatrics 1962;29:579-88.

4 Taubman B. Parental counselling compared with elimination of cows' milk or soy milk protein for the treatment of infant colic syndrome: a randomised trial. Pediatrics 1988;81: 756-61.

5 Miller JJ, McVeagh P, Fleet GH, Petocz P, Brand JC. Breath hydrogen excretion in infants with colic. Arch Dis Child 1989;64:725-9.

6 McKenzie SA. Breath hydrogen excretion in infants. Arch Dis Child 1989;64:1208.

7 Hunziker UA, Barr RG. Increased carrying reduces infant crying. Pediatrics 1986;77:641.

8 Carey WB. 'Colic'-primary excessive crying as an infantenvironment interaction. Pediatr Clin North Am 1984;31: 993-1005.

9 Rickert VI, Johnson CM. Reducing nocturnal awakening and crying episodes in infants and young children: a comparison between scheduled wakenings and systematic ignoring. Pediatrics 1988;81:203-12.

10 Taubman B. Clinical trial of the treatment of colic by modification of parent-infant interaction Pediatrics 1984;74: 998-1003.

11 Barbero GJ, Ringler D, Rose JA. Infantile gastrointestina disturbances: a pilot study and design for research. $A m \mathcal{J}$ Dis Child 1957:94:532.

12 Savilahanti E, Stahlberg M-R. Lactose tolerance in colicky infants. F Pediatr 1989;115:333-4.

13 Levine RA. Child rearing as cultural adaptation. In: Leiderman PH, Tulkin SR, Rosenfield A, eds. Culture and infancy: variations in the human experience. New York: Academic Press, 1977.

14 Brazelton TB. Infants and mothers. New York: Delta, 1983.

15 Pederson DR. The soothing effects of rocking as determined by the direction and frequency of movement. Canadian fournal of Behavioural Science 1975;7:237-43. 